\title{
On a Spacelike Line Congruence Which Has the Parameter Ruled Surfaces as Principal Ruled Surfaces
}

\author{
Ferhat Taş* and Rashad A. Abdel-Baky \\ (Communicated by Yusuf Yaylı)
}

\begin{abstract}
In this paper, we define spacelike line congruence, which has the parameter ruled surfaces as principal ruled surfaces, on the dual unit hyperbolic sphere $H_{+}^{2}$ in the dual Lorentzian 3-space $D_{1}^{3}$. We carry the obtained results to the 3-dimensional Minkowski space $E_{1}^{3}$ by means of the E. Study's dual line coordinates.
\end{abstract}

Keywords: Spacelike line congruence; Hamilton and Mannhiem formulae; Plücker conoid.

AMS Subject Classification (2010): Primary: 53A04; Secondary: 53A05; 53A17.

\section{Introduction}

In the Euclidean 3-space $\mathrm{E}^{3}$, a two-parameter set of lines is called a rectilinear line congruence (line congruence for short), where the normals to a surface constitute such a line congruence. Generally, the lines of a congruence are not normal to a surface. Hence, the line congruence of normals forms a special class; which is called normal line congruence. In this case, the normals along a principal curvature line constitute a developable ruled surface. Hence, the line congruence of normals forms a special class; which is called the normal line congruence (geometric optic). The lines of the line congruence which pass through a curve on the surface form a one-parameter family of lines in the space or ruled surface (parameter ruled surface). Nowadays, the differential geometry of line congruences has become relevant for practical applications, see for example $[6,7,8,11]$.

Rather unexpectedly dual numbers have been applied to study the motion of a line space; they seem even be the most appropriate apparatus for this end. In screw and dual number algebra, the E. Study's dual line coordinates conclude: The set of all oriented lines in Euclidean 3-space $E^{3}$ is in one-to-one correspondence with the set of points of the dual unit sphere in the dual 3-space $D^{3}$. Hence, the ruled surfaces and line congruence obtained by the motion of a line, depending on one and two parameters, respectively, were examined more easily, as reported in [1-4,11].

A large number of papers have been published in the literature which deal with line congruences in both Minkowski space and Euclidean space (See for instance Refs. $([1-4,10,12-16,19,20])$. The main interest of this paper is to introduce the E. Study's dual line coordinates in the dual Lorentzian 3-space $D_{1}^{3}$. As an application for the theory of line congruence, a spacelike line congruence which has the parameter ruled surfaces as principal ruled surfaces was introduced. In terms of this, the results can easily be transferred to the Minkowski 3-space $E_{1}^{3}$, and for them the geometric explanations are derived.

We hope that these results will be helpful to physicists and those studying general relativity theory. 


\section{Basic concepts}

In this section, we give a brief summary of the theory of dual numbers, dual Lorentzian vectors and E. Study's dual line coordinates. For more details, we refer to $[5,9,17,18]$. Let $E_{1}^{3}$ be the three-dimensional Minkowski space, that is, the three-dimensional real vector space $\mathbb{R}^{3}$ with the metric

$$
<d \mathbf{x}, d \mathbf{x}>=d x_{1}^{2}+d x_{2}^{2}-d x_{3}^{2},
$$

where $\left(x_{1}, x_{2}, x_{3}\right)$ denotes the canonical coordinates in $\mathbb{R}^{3}$. An arbitrary vector $\mathbf{x}$ of $E_{1}^{3}$ is said to be spacelike if $\langle\mathbf{x}, \mathbf{x}>>0$ or $\mathbf{x}=\mathbf{0}$, timelike if $\langle\mathbf{x}, \mathbf{x}\rangle<0$ and lightlike or null if $\langle\mathbf{x}, \mathbf{x}>=0$ and $\mathbf{x}=\mathbf{0}$. A timelike or light-like vector in $E_{1}^{3}$ is said to be causal. For $\mathbf{x} \in E_{1}^{3}$ the norm is defined by $\|\mathbf{x}\|=\sqrt{|\langle\mathbf{x}, \mathbf{x}\rangle|}$, then the vector $\mathbf{x}$ is called a spacelike unit vector if $\langle\mathbf{x}, \mathbf{x}\rangle=1$ and a timelike unit vector if $\langle\mathbf{x}, \mathbf{x}\rangle=-1$. Similarly, a regular curve in $E_{1}^{3}$ can locally be spacelike, timelike or null (lightlike), if all of its velocity vectors are spacelike, timelike or null (lightlike), respectively. For any two vectors $\mathbf{x}=\left(x_{1}, x_{2}, x_{3}\right)$ and $\mathbf{y}=\left(y_{1}, y_{2}, y_{3}\right)$ of $E_{1}^{3}$, the inner product is the real number $\langle\mathbf{x}, \mathbf{y}\rangle=x_{1} y_{1}+x_{2} y_{2}-x_{3} y_{3}$ and the vector product is defined by $\mathbf{x} \times \mathbf{y}=\left(\left(x_{2} y_{3}-x_{3} y_{2}\right),\left(x_{3} y_{1}-x_{1} y_{3}\right),-\left(x_{1} y_{2}-x_{2} y_{1}\right)\right)$.

\subsection{Dual Lorentzian 3-Space $\mathbf{D}_{1}^{3}$}

A dual number $A$ has the form $a+\varepsilon a^{*}$ where $a, a^{*}$ are real numbers. Here $\varepsilon$ is a dual unit subject to the rules $\varepsilon \neq 0, \varepsilon^{2}=0, \varepsilon .1=1 . \varepsilon=\varepsilon$. The set of dual numbers $D$ forms a commutative ring having the numbers $\varepsilon a^{*}\left(a^{*}\right.$ real $)$ as divisors of zero. $D$ is not a field. No number $\varepsilon a^{*}$ has an inverse in the algebra. But the other laws of the algebra of dual numbers are the same as of the complex numbers.

For all pairs $\left(\mathbf{a}, \mathbf{a}^{*}\right) \in E_{1}^{3} \times E_{1}^{3}$ the set

$$
D^{3}=\left\{\mathbf{A}=\mathbf{a}+\varepsilon \mathbf{a}^{*}, \varepsilon \neq 0, \varepsilon^{2}=0\right\},
$$

together with the Lorentzian inner product

$$
<\mathbf{A}, \mathbf{B}>=<\mathbf{a}, \mathbf{b}>+\varepsilon\left(<\mathbf{b}, \mathbf{a}^{*}>+<\mathbf{b}^{*}, \mathbf{a}>\right),
$$

forms the dual Lorentzian 3-space $D_{1}^{3}$. Thereby a point $\mathbf{A}=\left(A_{1}, A_{2}, A_{3}\right)^{t}$ has dual coordinates $A_{i}=\left(a_{i}+\varepsilon a_{i}^{*}\right) \in$ $D$. The norm is defined by

$$
<\mathbf{A}, \mathbf{A}>^{\frac{1}{2}}=:\|\mathbf{A}\|=\|\mathbf{a}\|\left(1+\varepsilon \frac{<\mathbf{a}, \mathbf{a}^{*}>}{\|\mathbf{a}\|^{2}}\right),
$$

In the dual Lorentzian 3-space $D_{1}^{3}$ the dual hyperbolic and dual Lorentzian unit spheres, respectively, are

$$
H_{+}^{2}=\left\{A \in D_{1}^{3} \mid A_{1}^{2}+A_{2}^{2}-A_{3}^{2}=-1, A_{3}>0\right\},
$$

and

$$
S_{1}^{2}=\left\{A \in D_{1}^{3} \mid A_{1}^{2}+A_{2}^{2}-A_{3}^{2}=1\right\}
$$

respectively. Therefore, the E. Study's dual line coordinates can be stated as follows: The dual unit spheres are shaped like a pair of conjugate hyperboloids. The common asymptotic cone represents the set of null lines, the ring shaped hyperboloid represents the set of spacelike lines, and the oval shaped hyperboloid forms the set of timelike lines, opposite points of each hyperboloid represent the pair of opposite vectors on a line (see Fig. 1).

i) Spacelike dual angle: Let $\mathbf{A}$ and $\mathbf{B}$ be spacelike dual vectors in $D_{1}^{3}$ that span a spacelike dual vector subspace; then we have $\mid\langle\mathbf{A}, \mathbf{B}>|=\|\mathbf{A}\|\|\mathbf{B}\|$, and hence, there is a unique dual number $\Theta=\vartheta+\varepsilon \vartheta^{*} \geq 0$ such that $<\mathbf{A}, \mathbf{B}>=\|\mathbf{A}\|\|\mathbf{B}\| \cos \Theta$. This number is called the spacelike dual angle between the dual vectors $\mathbf{A}$ and B.

ii) Central dual angle: Let $\mathbf{A}$ and $\mathbf{B}$ be spacelike dual vectors in $D_{1}^{3}$ that span a timelike dual vector subspace; then we have $|<\mathbf{A}, \mathbf{B}>|>\|\mathbf{A}\|\|\mathbf{B}\|$, and hence, there is a unique dual number $\Theta=\vartheta+\varepsilon \vartheta^{*} \geq 0$ such that $<\mathbf{A}, \mathbf{B}>=\|\mathbf{A}\|\|\mathbf{B}\| \cosh \Theta$. This dual number is called the central dual angle between the dual vectors $\mathbf{A}$ and B.

iii) Lorentzian timelike dual angle: Let $\mathbf{A}$ be spacelike dual vector and $\mathbf{B}$ be timelike dual vector in $D_{1}^{3}$. Then there is a unique dual number $\Theta=\vartheta+\varepsilon \vartheta^{*} \geq 0$ such that $\langle\mathbf{A}, \mathbf{B}>=\|\mathbf{A}\|\|\mathbf{B}\| \sinh \Theta$. This dual number is called the Lorentzian timelike dual angle between the dual vectors $\mathbf{A}$ and $\mathbf{B}$. 


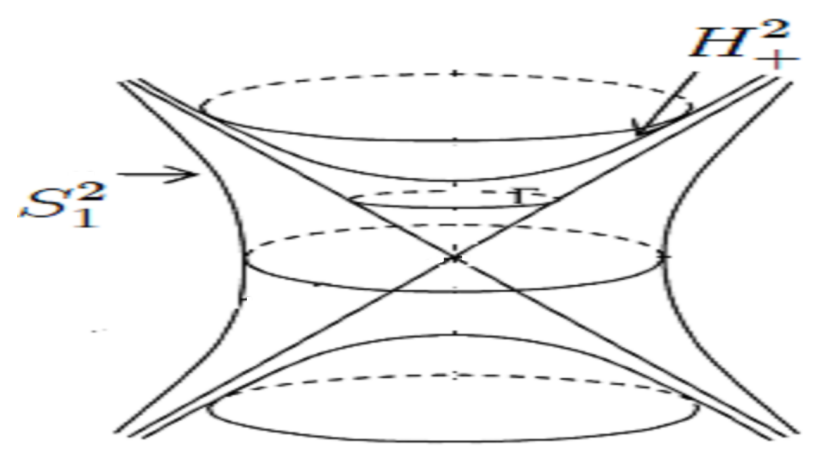

Figure 1. The dual hyperbolic and dual Lorentzian unit spheres.

\section{Spacelike line Congruence}

According to the E. Study's dual line coordinates, a space-like line congruence can be represented by a spacelike dual unit vector which is depending on two real parameters $u_{1}$, and $u_{2}$ as follows:

$$
\mathbf{A}\left(u_{1}, u_{2}\right)=\mathbf{a}\left(u_{1}, u_{2}\right)+\varepsilon \mathbf{a}^{*}\left(u_{1}, u_{2}\right) .
$$

A relation such as $\rho\left(u_{1}, u_{2}\right)=0$, between the parameters restricts (3.1) to one-parameter set of straight lines, i.e. a ruled surface in the congruence. The equation $u_{2}=c_{2}$ (real constant) determines a ruled surface in the congruence or a dual curve on the dual unit spheres (pair of conjugate hyperboloids). When the constant $c_{2}$ is varied a family of dual curves is generated, which we call the $u_{1}$-parameter curves, since along any one of them $u_{1}$-alone varies. Similarly, we have a family of $u_{1}=c_{1}$ (real constant) parameter curves. It follows from our insistence on the one-to-one correspondence between pairs of parameter curves and the ruled surfaces in the congruence, that each member of one family of dual curves cuts each member of the other once and only once. Any two families of dual curves satisfying the above conditions will provide us with a principal ruled surfaces which could be used to study the congruence. Thus, we may choose $\mathbf{A}_{u_{1}}=\left(\partial \mathbf{A} / \partial u_{1}\right)$ is a timelike (resp. a spacelike) dual vector in direction in which $u_{1}$-alone varies, i.e. is tangent to the $u_{1}$-parameter dual curve, which implies that $\mathbf{A}_{u_{2}}$ is spacelike dual vector (similar procedures can be applied). The displacement $d \mathbf{A}$ from the point $\left(u_{1}, u_{2}\right)$ to the point $\left(u_{1}+d u_{1}, u_{2}+d u_{2}\right)$ is:

$$
\left.\begin{array}{l}
d S^{2}=<d \mathbf{A}, d \mathbf{A}>=<\mathbf{A}_{u_{1}} d u_{1}+\mathbf{A}_{u_{2}} d u_{2}, \mathbf{A}_{u_{1}} d u_{1}+\mathbf{A}_{u_{2}} d u_{2}> \\
\quad=-E d u_{1}^{2}+2 F d u_{1} d u_{2}+G d u_{2}^{2}
\end{array}\right\}
$$

where

$$
-\left\|\mathbf{A}_{u_{1}}\right\|^{2}:=E=e+\varepsilon e^{*},\left\langle\mathbf{A}_{u_{1}}, \mathbf{A}_{u_{2}}>:=F=f+\varepsilon f^{*},\left\|\mathbf{A}_{u_{2}}\right\|^{2}:=G=g+\varepsilon g^{*} .\right.
$$

The quantities $E=e+\varepsilon e^{*}, \quad F=f+\varepsilon f^{*}, G=g+\varepsilon g^{*}$ are important because the distance between neighboring dual points may be expressed in term of them. Then by calculating the real and dual parts, we get:

$$
\left.\begin{array}{l}
I=d s^{2}=<d \mathbf{a}, d \mathbf{a}>=e d u_{1}^{2}+2 f d u_{1} d u_{2}+g d u_{2}^{2} \\
I I=2 d s d s^{*}=2<d \mathbf{a}, d \mathbf{a}^{*}>=e^{*} d u_{1}^{2}+2 f^{*} d u_{1} d u_{2}+g^{*} d u_{2}^{2} .
\end{array}\right\}
$$

The forms $I$ and $I I$ are called the first and second fundamental forms of the congruence: Here we exclude the case where the coefficients of $I I$ and $I$ are proportional, i.e. the congruence is isotropic congruence.

Now suppose that $\left\|\mathbf{a}_{u_{1}} \times \mathbf{a}_{u_{2}}\right\|^{2}=e g+f^{2} \neq 0$, i.e. the congruence is not a cylindrical congruence. The distribution parameter of a ruled surface in the congruence is given by

$$
\lambda=\frac{I I}{2 I}=\frac{1}{2}\left(\frac{-e^{*} d u_{1}^{2}+2 f^{*} d u_{1} d u_{2}+g^{*} d u_{2}^{2}}{-e d u_{1}^{2}+2 f d u_{1} d u_{2}+g d u_{2}^{2}}\right) .
$$

The function $\lambda$ is a function of the ratio $\left(d u_{1} / d u_{2}\right)$, so it varies with the value of this ratio. There are two values of this ratio for which $\lambda$ is a maximum or minimum. These are obtained by equating to zero the derivatives of $\lambda$ with respect to the ratio. This leads to the equation: 


$$
\left(e f^{*}-e^{*} f\right) d u_{1}^{2}+\left(e g^{*}-e^{*} g\right) d u_{1} d u_{2}+\left(g f^{*}-f g^{*}\right) d u_{2}^{2}=0 .
$$

Let the parameter ruled surfaces are taken as principal ruled surfaces. This must be equivalent to $d u_{1} d u_{2}=0$, hence Eq. (3.5) shows that:

$$
e f^{*}-e^{*} f=0, g f^{*}-f g^{*}=0,
$$

therefore, since the coefficients of $I$, and $I I$ are not proportional, we must have:

$$
f=<\mathbf{a}_{u_{1}}, \mathbf{a}_{u_{1}}>=0, f^{*}=<\mathbf{a}_{u_{1}}^{*}, \mathbf{a}_{u_{2}}>+<\mathbf{a}_{u_{2}}^{*}, \mathbf{a}_{u_{1}}>=0 .
$$

Hence, the necessary and sufficient condition that the principal ruled surfaces are represented by orthogonal curves is given:

$$
F=f+\varepsilon f^{*}=0 .
$$

\subsection{The Blaschke frames}

As for surfaces, through every line in the congruence there pass two principal surfaces whose images on the dual Lorentzian unit sphere we take as parametric curves. For this special system we assume that $u_{1}$-and $u_{2}$ dual curves of $\mathbf{A}\left(u_{1}, u_{2}\right)$ are principal ruled surfaces, i.e., the elements $f$ and $f^{*}$ of the first and second fundamental forms vanish identically $\left(f=f^{*}=0\right)$. Consider now the dual unit vectors $\mathbf{A}_{12}=\mathbf{A}_{12}\left(u_{1}, u_{2}\right)$, and $\mathbf{A}_{22}=\mathbf{A}_{22}\left(u_{1}, u_{2}\right)$, are the tangents of the parametric dual curves $u_{2}=$ const., and $u_{1}=$ const., respectively, at any regular point, then we have:

$$
\mathbf{A}_{12}=\frac{\mathbf{A}_{u_{1}}}{\sqrt{\left|<\mathbf{A}_{u_{1}}, \mathbf{A}_{\mathbf{u}_{\mathbf{1}}}>\right|}}=\frac{\mathbf{A}_{u_{1}}}{\sqrt{E}} \text {, and } \mathbf{A}_{22}=\frac{\mathbf{A}_{u_{2}}}{\sqrt{<\mathbf{A}_{u_{2}}, \mathbf{A}_{\mathbf{u}_{\mathbf{2}}}>}}=\frac{\mathbf{A}_{u_{1}}}{\sqrt{G}},
$$

which are invariants vector functions on the congruence. Hence, we fix $\operatorname{det}\left(\mathbf{A}, \mathbf{A}_{12}, \mathbf{A}_{22}\right)=+1$ and consequently

$$
\left.\begin{array}{l}
\mathbf{A}=\mathbf{A}_{12} \times \mathbf{A}_{22}, \mathbf{A}_{12}=\mathbf{A} \times \mathbf{A}_{22}, \mathbf{A}_{22}=\mathbf{A} \times \mathbf{A}_{12}, \\
-\left\|\mathbf{A}_{12}\right\|^{2}=\left\|\mathbf{A}_{22}\right\|^{2}=\|\mathbf{A}\|^{2}=1 .
\end{array}\right\}
$$

The dual arc-lengths, corresponding to the principal surfaces, are obtained by substituting first $d u_{2}=0$, then $d u_{1}=0$ into Eq. (3.2), that:

$$
d S_{1}:=d s_{1}+\varepsilon d s_{1}^{*}=P_{1} d u_{1}, \quad d S_{2}:=d s_{2}+\varepsilon d s_{2}^{*}=P_{2} d u_{2},
$$

where $P_{1}=p_{1}+\varepsilon p_{1}^{*}=\sqrt{E}$, and $P_{2}=p_{2}+\varepsilon p_{2}^{*}=\sqrt{G}$. The distribution parameters of the principal ruled surfaces $\mathbf{A}\left(u_{1}, c_{2}\right)$, and $\mathbf{A}\left(c_{1}, u_{2}\right)$, respectively, are:

$$
\lambda_{1}:=\frac{d s_{1}^{*}}{d s_{1}}=\frac{e^{*}}{2 e}, \quad \lambda_{2}:=\frac{d s_{2}^{*}}{d s_{2}}=\frac{g^{*}}{2 g} .
$$

According to the elements of spherical kinematics, the motion of the frame $\left\{\mathbf{A}, \mathbf{A}_{12}, \mathbf{A}_{22}\right\}$ at any instant is a rotation around the Darboux vector $\mathbf{D}_{1}$ of this frame, i.e.

$$
\frac{\partial}{\partial S_{1}}\left(\begin{array}{l}
\mathbf{A} \\
\mathbf{A}_{12} \\
\mathbf{A}_{22}
\end{array}\right)=\left(\begin{array}{lll}
0 & 1 & 0 \\
1 & 0 & -\Sigma_{1} \\
0 & -\Sigma_{1} & 0
\end{array}\right)\left(\begin{array}{l}
\mathbf{A} \\
\mathbf{A}_{12} \\
\mathbf{A}_{22}
\end{array}\right)=\mathbf{D}_{1} \times\left(\begin{array}{l}
\mathbf{A} \\
\mathbf{A}_{12} \\
\mathbf{A}_{22}
\end{array}\right),
$$

where

$$
\mathbf{D}_{1}=-\Sigma_{1} \mathbf{A}-\mathbf{A}_{22} .
$$

$\Sigma_{1}=\sigma_{1}+\varepsilon \sigma_{1}^{*}=\frac{E_{u_{2}}}{2 E \sqrt{G}}$ is the geodesic curvature of the dual curves $u_{2}=c_{2}$. Similarly, the derivative formula of the Blaschke frame of the dual curves $u_{1}=$ const., with tangent $\mathbf{A}_{22}$ is:

$$
\frac{\partial}{\partial S_{2}}\left(\begin{array}{l}
\mathbf{A} \\
\mathbf{A}_{12} \\
\mathbf{A}_{22}
\end{array}\right)=\left(\begin{array}{lll}
0 & 0 & 1 \\
0 & 0 & \Sigma_{2} \\
-1 & \Sigma_{2} & 0
\end{array}\right)\left(\begin{array}{l}
\mathbf{A} \\
\mathbf{A}_{12} \\
\mathbf{A}_{22}
\end{array}\right)=\mathbf{D}_{2} \times\left(\begin{array}{l}
\mathbf{A} \\
\mathbf{A}_{12} \\
\mathbf{A}_{22}
\end{array}\right) .
$$

where

$$
\mathbf{D}_{2}=\Sigma_{2} \mathbf{A}-\mathbf{A}_{12}
$$


Also $\Sigma_{2}=\sigma_{2}+\varepsilon \sigma_{2}^{*}=\frac{G_{u_{1}}}{2 G \sqrt{E}}$ has the same meaning as in Eq. (3.13), for the curves $u_{1}=c_{1}$. In other words, dual geodesic curvatures of the dual curves $\mathbf{A}\left(u_{1}, c_{2}\right), \mathbf{A}\left(c_{1}, u_{2}\right)$, respectively, are:

$$
\Sigma_{i}=\sigma_{i}+\varepsilon \sigma_{i}^{*}=\operatorname{det}\left(\mathbf{A}, \frac{d \mathbf{A}}{d S_{i}}, \frac{d^{2} \mathbf{A}}{d S_{i}^{2}}\right),(i=1,2) .
$$

\subsection{Minkowski formulae of surfaces theory}

In this paragraph, we aim to give resemblance formulas of the well-known formulas of surfaces theory into line congruence in dual Lorentzian 3-space $D_{1}^{3}$. For this purpose, consider $u_{i}=u_{i}(t),(i=1,2)$ as functions of the real parameter $t$. Then $\mathbf{A}=\mathbf{A}\left(u_{1}(t), u_{2}(t)\right)$ represents a spacelike parameter ruled surface in the congruence. The dual vector $\mathbf{A}_{t}=(\partial \mathbf{A} / \partial t)$ is tangent to this dual curve;

$$
\mathbf{A}_{t}=\mathbf{A}_{u_{1}} \frac{d u_{1}}{d t}+\mathbf{A}_{u_{2}} \frac{d u_{2}}{d t} .
$$

If $<\mathbf{A}_{t}, \mathbf{A}_{t}>\neq 0$, then we have a dual unit vector

$$
\mathbf{A}_{2}=\frac{\mathbf{A}_{t}}{\left\|\mathbf{A}_{t}\right\|}=\frac{1}{P}\left(\mathbf{A}_{u_{1}} \frac{d u_{1}}{d t}+\mathbf{A}_{u_{2}} \frac{d u_{2}}{d t}\right)
$$

where $P:=p+\varepsilon p^{*}=\left\|\mathbf{A}_{u_{1}} \frac{d u_{1}}{d t}+\mathbf{A}_{u_{2}} \frac{d u_{2}}{d t}\right\|=\sqrt{E G}$. Hence, the dual arc length of the dual curve $\mathbf{A}(t)=$ $\mathbf{A}\left(u_{1}(t), u_{2}(t)\right)$ is given by

$$
d S=P d t .
$$

In order to research the properties of $\mathbf{A}(t)=\mathbf{A}\left(u_{1}(t), u_{2}(t)\right)$, the Blaschke frame relative to $\mathbf{A}(t)$ will be defined as the frame of which this line and the central normal $\mathbf{A}_{2}$ to the ruled surface at the central point of $\mathbf{A}$ are two edges. The third edge $\mathbf{A}_{3}$ is the central tangent to the ruled surface $\mathbf{A}(t)$. The frame $\left\{\mathbf{A}_{1}=\mathbf{A}(t), \mathbf{A}_{2}(t)=\right.$ $\left.\frac{\mathbf{A}_{1}^{\prime}}{\left\|\mathbf{A}_{1}^{\prime}\right\|}, \mathbf{A}_{3}(t)=\mathbf{A}_{1} \times \mathbf{A}_{2} ;\left({ }^{\prime}=\frac{d}{d t}\right)\right\}$ is called Blaschke frame. If $\mathbf{A}_{2}$ is a spacelike (timelike) dual unit vector while $\mathbf{A}_{3}$ is a timelike (spacelike) dual unit vector (similar procedures can be applied). Then we have:

$$
\begin{gathered}
<\mathbf{A}_{1}, \mathbf{A}_{1}>=<\mathbf{A}_{2}, \mathbf{A}_{2}>=-<\mathbf{A}_{3}, \mathbf{A}_{3}>=1, \\
<\mathbf{A}_{1}, \mathbf{A}_{2}>=<\mathbf{A}_{2}, \mathbf{A}_{3}>=<\mathbf{A}_{3}, \mathbf{A}_{1}>=0, \\
\mathbf{A}_{1} \times \mathbf{A}_{2}=-\mathbf{A}_{3}, \mathbf{A}_{1} \times \mathbf{A}_{3}=-\mathbf{A}_{2}, \mathbf{A}_{2} \times \mathbf{A}_{3}=\mathbf{A}_{1},
\end{gathered}
$$

The dual arc length derivative of the Blaschke frame is governed by the relations:

$$
\frac{d}{d S}\left(\begin{array}{c}
\mathbf{A}_{1} \\
\mathbf{A}_{2} \\
\mathbf{A}_{3}
\end{array}\right)=\left(\begin{array}{lll}
0 & 1 & 0 \\
-1 & 0 & \Sigma \\
0 & \Sigma & 0
\end{array}\right)\left(\begin{array}{c}
\mathbf{A}_{1} \\
\mathbf{A}_{2} \\
\mathbf{A}_{3}
\end{array}\right)=\mathbf{D} \times\left(\begin{array}{c}
\mathbf{A}_{1} \\
\mathbf{A}_{2} \\
\mathbf{A}_{3}
\end{array}\right),
$$

where

$$
\mathbf{D}=-\Sigma \mathbf{A}_{1}+\mathbf{A}_{3}
$$

$\Sigma(t)=\sigma+\varepsilon \sigma^{*}$ is the dual geodesic curvature of the dual curvature of the dual curve $\mathbf{A}\left(u_{1}(t), u_{2}(t)\right)$. It is easily seen from the last two equations that

$$
\Sigma=\sigma+\varepsilon \sigma^{*}=\frac{\operatorname{det}\left(\mathbf{A}, \mathbf{A}_{t}, \mathbf{A}_{t t}\right)}{\left\|\mathbf{A}_{t}\right\|^{3}}=\operatorname{det}\left(\mathbf{A}, \frac{d \mathbf{A}}{d S}, \frac{d^{2} \mathbf{A}}{d S^{2}}\right) .
$$

Referring to the congruence (3.1), since $\mathbf{A}_{t}$ is tangent of the dual curve $\mathbf{A}\left(u_{1}(t), u_{2}(t)\right)$, then Eq. (3.19) rewritten as

$$
\mathbf{A}_{2}=\frac{d S_{1}}{d S} \mathbf{A}_{12}+\frac{d S_{2}}{d S} \mathbf{A}_{22},
$$

in view of Eqs. (3.9), and (3.11). So, we can find a dual angle $\Phi=\varphi+\varepsilon \varphi^{*}$ such that (Fig. 2):

$$
\mathbf{A}_{2}=\sinh \Phi \mathbf{A}_{12}+\cosh \Phi \mathbf{A}_{22},
$$

where

$$
\begin{gathered}
d S^{2}=-d S_{1}^{2}+d S_{2}^{2}, \\
\sinh \Phi=\frac{d S_{1}}{d S}=\sqrt{E} \frac{d u_{1}}{d S}, \cosh \Phi=\frac{d S_{2}}{d S}=\sqrt{G} \frac{d u_{2}}{d S} .
\end{gathered}
$$


Consequently, by separating the real and dual parts of Eqs. (3.27), with attention of Eqs. (3.4) and (3.12), we get:

$$
\left.\begin{array}{l}
\lambda=-\lambda_{1} \sinh ^{2} \varphi+\lambda_{2} \cosh ^{2} \varphi, \\
\varphi^{*}=\left(\frac{\lambda_{1}-\lambda_{2}}{2}\right) \sinh 2 \varphi .
\end{array}\right\}
$$

These formulas are Minkowski versions of the Hamilton and Mannhiem formulae in Euclidean 3-space, respectively [1-4]. The surface described by $\varphi^{*}$ in Eqs. (3.28) is the Minkowski version of the Plücker conoid in Euclidean 3-space. The parametric form can also be given in terms of point coordinates. We may choose $\mathbf{A}$ is coincident with the $y$-axis of a fixed Lorentzian frame (oxyz), while the position of the dual unit vector $\mathbf{A}_{2}$ is given by angle $\varphi$ and distance $\varphi^{*}$ along the positive $y$-axis. The edges $\mathbf{A}_{22}$ and $\mathbf{A}_{12}$ can be selected in sense of $z$ and $x$-axes, respectively, as depicted in Fig. 2. Let $\mathbf{L}$ denote a point on this surface, it is possible to have the following point coordinates

$$
M: \mathbf{L}(\varphi, \mu)=\varphi^{*}\left(\begin{array}{l}
0 \\
1 \\
0
\end{array}\right)+\mu\left(\begin{array}{l}
\sinh \varphi \\
0 \\
\cosh \varphi
\end{array}\right)=\left(\begin{array}{c}
\mu \sinh \varphi \\
\left(\frac{\lambda_{1}-\lambda_{2}}{2}\right) \sinh 2 \varphi \\
\mu \cosh \varphi
\end{array}\right), \mu \in \mathbb{R} .
$$

We choose $\lambda_{1}-\lambda_{2}=1$, this parametrization defines a non-developable timelike ruled surface, which is called a helicoid of the 2nd kind; $\lambda_{1}-\lambda_{2}=1, \varphi \in[-1,1], \mu \in[-7,7]$ (see Fig. 3). If $(x, y, z)$ are the coordinates of $M$, then Eq. (3.29) yields:

$$
M: y=\frac{\beta x z}{z^{2}-x^{2}},
$$

where $\beta=\lambda_{1}-\lambda_{2}$. It is interesting to note that it is a third -order polynomial in the coordinates $x, y$ and $z$.

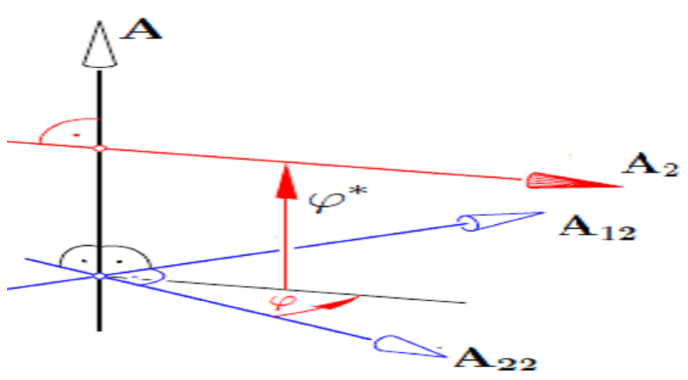

Figure 2. $\mathbf{A}_{2}=\sinh \Phi \mathbf{A}_{1} 2+\cosh \Phi \mathbf{A}_{2} 2$

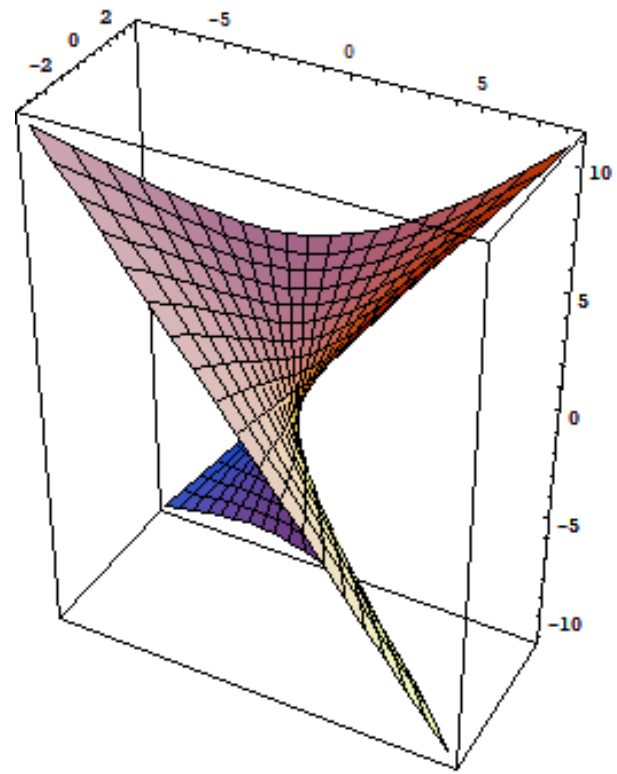

Figure 3. Helicoid of the 2nd kind

Differentiating Eq. (3.26), and using the formula

$$
\frac{d \mathbf{A}_{2}}{d S}=\left(\frac{\partial \mathbf{A}_{2}}{\partial S_{1}} \frac{d S_{1}}{d S}+\frac{\partial \mathbf{A}_{2}}{\partial S_{2}} \frac{d S_{2}}{d S}\right),
$$

we get:

$$
\begin{aligned}
\frac{d \mathbf{A}_{2}}{d S}= & \left(\frac{\partial \mathbf{A}_{12}}{\partial S_{2}}+\frac{\partial \mathbf{A}_{22}}{\partial S_{1}}\right) \cosh \Phi \sinh \Phi+\frac{\partial \mathbf{A}_{12}}{\partial S_{1}} \sinh ^{2} \Phi+\frac{\partial \mathbf{A}_{22}}{\partial S_{2}} \cosh ^{2} \Phi \\
& +\left(\mathbf{A}_{12} \cosh \Phi+\mathbf{A}_{22} \sinh \Phi\right) \frac{d \Phi}{d S},
\end{aligned}
$$

where, according to Eqs. (3.21),

$$
-\mathbf{A}_{3}=\cosh \Phi \mathbf{A}_{12}+\sinh \Phi \mathbf{A}_{22} .
$$


Hence, we obtain:

$$
-\mathbf{A}+\Sigma \mathbf{A}_{3}=\left(\Sigma_{1} \sinh \Phi-\Sigma_{2} \cosh \Phi-\frac{d \Phi}{d S}\right) \mathbf{A}_{3}-\mathbf{A}
$$

or finally

$$
\Sigma=\Sigma_{1} \sinh \Phi-\Sigma_{2} \cosh \Phi-\frac{d \Phi}{d S} .
$$

This formula is the same nature of J. Liouvile formula in surface theory in Euclidean 3-space $E^{3}$.

Theorem 3.1. In the congruence $\mathbf{A}\left(u_{1}, u_{2}\right)$, there exists a relation among the dual Darboux vectors of the Blaschke frames of the ruled surfaces $\mathbf{A}(t)=\mathbf{A}\left(u_{1}(t), u_{2}(t)\right), \mathbf{A}\left(u_{1}\right)=\mathbf{A}\left(u_{1}, c_{2}\right)$, and $\mathbf{A}\left(u_{2}\right)=\mathbf{A}\left(c_{1}, u_{2}\right)$. More explicitly, we have:

$$
\mathbf{D}=\sinh \Phi \mathbf{D}_{1}+\cosh \Phi \mathbf{D}_{2}+\left(\frac{d \Phi}{d S}\right) \mathbf{A}_{1} .
$$

Proof. Substituting Eq. (3.33) into Eq. (3.23) we may write D as:

$$
-\mathbf{D}=\Sigma \mathbf{A}_{1}+\cosh \Phi \mathbf{A}_{12}+\sinh \Phi \mathbf{A}_{22},
$$

or, because of Eqs. (3.14), and (3.16):

$$
-\mathbf{D}=\left(\Sigma-\Sigma_{1} \sinh \Phi+\Sigma_{2} \cosh \Phi-\frac{d \Phi}{d S}\right) \mathbf{A}_{1}+\cosh \Phi \mathbf{D}_{2}+\sinh \Phi \mathbf{D}_{1} .
$$

Taking into account the geodesic curvature $\Sigma$ as per Eq. (3.34) into Eq. (3.36), the relation is proved.

We intend now to give a geometrical interpretation of J. Liouvile formula. For this purpose, making use of Eqs. (3.27) into Eq. (3.34), then we have:

$$
\Sigma d S=\Sigma_{1} \sqrt{E} d u_{1}-\Sigma_{2} \sqrt{G} d u_{2}-d \Phi .
$$

We found, by application of Green theorem, that:

$$
\oint \Sigma d S=-\iint\left(\frac{\partial}{\partial u_{1}}\left(\Sigma_{2} \sqrt{G}\right)+\frac{\partial}{\partial u_{2}}\left(\Sigma_{1} \sqrt{E}\right)\right) d u_{1} d u_{2}-\oint d \Phi .
$$

The Gaussian curvature of the dual hyperbolic sphere $H_{+}^{2}$, in Eq. (2.4), is given by:

$$
-1=\frac{1}{\sqrt{E G}}\left(\frac{\partial}{\partial u_{1}}\left(\Sigma_{2} \sqrt{G}\right)+\frac{\partial}{\partial u_{2}}\left(\Sigma_{1} \sqrt{E}\right)\right) .
$$

So that, we obtain as a result the formula

$$
\oint \Sigma d S=\iint d A-\oint d \Phi
$$

where $d A=\sqrt{E G} d u_{1} d u_{2}$ is the dual area on $H_{+}^{2}$ enclosed by the dual curve $\mathbf{A}=\mathbf{A}\left(u_{1}(t), u_{2}(t)\right)$. The formula (3.40) is resemblance to the Gauss-Bonnet formula in surfaces theory. This formula may be reduces to

$$
\oint \Sigma d S=\iint d S_{1} d S_{2}-\oint d \Phi
$$

in view of Eqs. (3.27). We replace of the distribution parameters $\lambda_{1}$ and $\lambda_{2}$, as per Eqs. (3.12) into Eq. (3.41), thus obtaining:

$$
\begin{aligned}
\oint \Sigma d S & =\iint\left(1+\varepsilon \lambda_{1}\right)\left(1+\varepsilon \lambda_{2}\right) d s_{1} d s_{2}-\oint d \Phi \\
& =a_{r}-2 \pi+\varepsilon\left(\iint 2 h d s_{1} d s_{2}-\varphi^{*}\right)
\end{aligned}
$$


where $a_{r}$ is the real part of element area on $H_{+}^{2}$ described by the real spherical curve $\mathbf{a}=\mathbf{a}(t)$, and $h=$ $\left(\lambda_{1}+\lambda_{2}\right) / 2$ is the mean distribution parameter of the congruence $\mathbf{A}\left(u_{1}, u_{2}\right)$. On the other side, the dual angle of the pitch of the closed surface $\mathbf{A}=\mathbf{A}\left(u_{1}(t), u_{2}(t)\right)$ is [8]:

$$
\Lambda_{a}=\mu_{a}-\varepsilon \Gamma_{a}=-\oint \Sigma d S,
$$

where $\mu_{a}$ is the angle of pitch and $\Gamma_{a}$ is the pitch of the closed ruled surface $\mathbf{A}=\mathbf{A}\left(u_{1}(t), u_{2}(t)\right)$. When Eq. (3.43) is applied to Eq. (3.42) we immediately find that:

$$
\mu_{a}-\varepsilon \Gamma_{a}=2 \pi-a_{r}-\varepsilon\left(\iint 2 h d s_{1} d s_{2}-\varphi^{*}\right),
$$

Now, separating Eqs. (3.44), we find the real parts

$$
\mu_{a}=2 \pi-a_{r},
$$

and the dual parts

$$
\Gamma_{a}=\iint 2 h d s_{1} d s_{2}-\varphi^{*} .
$$

Therefore, we can write J. Liouvile formula as:

$$
\begin{aligned}
\Lambda_{a} & =\oint\left(-\Sigma_{1} d S_{1}+\Sigma_{2} d S_{2}\right)+2 \pi+\varepsilon \varphi^{*} \\
& =-\oint \Sigma_{1} d S_{1}+\oint \Sigma_{2} d S_{2}+2 \pi+\varepsilon \varphi^{*} .
\end{aligned}
$$

Then, from the relations above

$$
\Lambda_{a}=\Lambda_{a 1}-\Lambda_{a 2}+2 \pi+\varepsilon \varphi^{*},
$$

are fund. Here, $\Lambda_{a 1}$, and $\Lambda_{a 2}$ are the dual angels of pitch of the principal ruled surfaces $\mathbf{A}\left(u_{1}\right)=\mathbf{A}\left(u_{1}, c_{2}\right)$, and $\mathbf{A}\left(u_{2}\right)=\mathbf{A}\left(c_{1}, u_{2}\right)$, respectively.

\section{References}

[1] Abdel-Baky, R.A.; The relation among Darboux vectors of ruled surfaces in a line congruence, Riv. Mat., Univ. Parama, (1997), (5)6, pp.201211.

[2] Abdel-Baky, R.A. On the congruences of the tangents to a surface, Anz. Osterreich. Akad. Wiss. Math.-Natur. Kl., (1999), $136: 9-18$.

[3] Abdel-Baky, R.A. . On instantaneous rectilinear congruences, J. Geom. Graph., (2003), 7(2): 129-135.

[4] Abdel-Baky, R. A. On a line congruence which has the parameter ruled surfaces as principal ruled surfaces, Applied mathematics and computation, 151.3 (2004): 849-862.

[5] Blaschke, W.; Vorlesungen uber Differential Geometrie, Bd 1, Dover Publications, New York, pp.260-277, 1945.

[6] Bottema, O. and Roth, B. Theoretical Kinematics, North-Holland Press, New York, 1979.

[7] Odehnal, B. and Pottmann, H. Computing with discrete models of ruled surfaces and line congruences, Proceedings of the 2nd workshop on computational kinematics, Seoul 2001.

[8] Odehnal, B. Geometric Optimization Methods for Line Congruences, Ph. D. Thesis, Vienna University of Technology, 2002.

[9] O’Neil, B. Semi-Riemannian Geometry with Applications to Relativity. Academic Press, London, 1983.

[10] Papadopoulou, D. and Koltsaki, P. Triply orthogonal line congruences with common middle surface, J. Geom. Graph., (2010), $14(1)$ : 45-58.

[11] Pottman, H. and Wallner, J. Computational Line Geometry, Springer-Verlag, Berlin, Heidelberg, 2001.

[12] F. Taş, 2016, "On the Computational Line Geometry," Ph.D. thesis, Institute of Graduate Studies in Science and Engineering, Istanbul University, Istanbul.

[13] F. Taş, O. Gürsoy, 2018, On the Line Congruences, International Electronic Journal of Geometry, Vol. 11(2), pp. 47-53.

[14] Tsagas, Gr. "On the rectilinear congruences of Lorentz manifold establishing an area preserving representation." Tensor, NS 47 (1988): 127-139.

[15] Tunahan, T., Nurai, Y. and Nihat, A. On pseudohyperbolic space motions, Turk J Math., (2015), 39: 750-762, TUBITAK doi:10.3906/mat1503-37.

[16] Uğurlu, H.H.; Çalışkan, A. The Study Mapping for Directed Space-Like and Time-Like in Minkowski 3-Space R13. Math. Comput. Appl., (1996), 1, 142-148.

[17] Veldkamp, G.R.; On the use of dual numbers, vectors, and matrices in instantaneous spatial kinematics, Mech. and Mach. Theory, (1976), V.11, pp.141-156.

[18] Yaglom, I.M. A Simple Non-Euclidean Geometry and Its Physical Basis. Springer-Verlag, New York, 1979.

[19] Yayli, Y, Caliskan A. and Ugurlu, H.H. The E study maps of circles on dual hyperbolic and Lorentzian unit spheres $\mathrm{H}_{0}^{2}$ and $\mathrm{S}_{1}^{2}$, Mathematical Proceedings of the Royal Irish Academy, (2002), 102A: 37-47.

[20] Zhong, H. and Hual, Li, L. A kind of rectilinear congruences in the Minkowski 3-Space, J. of Mathematical Research \& Exposition, Nov., 2008, Vol. 28, No. 4 pp. 911-918. 


\section{Affiliations}

FERHAT TAŞ

AdDRESs: Department of Mathematics, Faculty of Science, Istanbul University, 34134, Istanbul, Turkey E-MAIL: tasf@istanbul.edu.tr

ORCID ID : orcid.org/0000-0001-5903-2881

RASHAD A. ABDEL-BAKY

ADDRESS: 1) Department of Mathematics, Sciences Faculty for Girls, King Abdulaziz University, Jeddah 21352, SAUDI ARABIA

2) Department of Mathematics, Faculty of Science, University of Assiut, Assiut 71516 , EGYPT E-MAIL: rbaky@live.com

ORCID ID : orcid.org/0000-0001-7016-9280 